\title{
EFEITOS DA MANIPULAÇÃO NA LOMBALGIA CRÔNICA
}

\section{EFFECTS OF MANIPULATION ON CHRONIC LOW BACK PAIN}

\author{
Maria Ida Barbosa Lima; \\ Acadêmica do $9^{\circ}$ período do Curso de Fisioterapia da-UNIPAC. \\ mariaidablima@hotmail.com \\ Thalia Almeida Viana; \\ Acadêmica do $9^{\circ}$ período do Curso de Fisioterapia da-UNIPAC. \\ thaliafisio97@hotmail.com \\ Matteus Cordeiro de Sá \\ Fisioterapeuta- Especialista em Fisioterapia Traumato-Ortopédica. \\ matteuscordeirodesá@gmail.com
}

\section{RESUMO}

A lombalgia crônica é uma dor contínua na região lombar que não apresenta melhora após três meses ou mais. Tem uma alta prevalência na população e é um dos principais problemas de saúde ocupacional. Utiliza-se frequentemente a manipulação como tratamento, que consiste na aplicação de técnicas com as mãos, sobre o corpo do paciente, com o objetivo de promover o retorno à função normal, destacando-se por reestabelecer a mobilidade articular, promover analgesia e alterar a atividade muscular. Esse artigo é uma revisão na literatura científica referente à técnica de manipulação na dor lombar crônica. Foram pesquisadas as principais bases de dados e considerado artigos de 2004 até o momento, além de livros da área. Os estudos são controversos, alguns demonstram a eficácia no alívio da dor logo após a aplicação, já alguns estudos não foram satisfatórios. A terapia com mais sessões pareceu trazer benefícios. Há a falta de estudos com mais pacientes e mais profissionais envolvidos, porém a manipulação é uma técnica bastante utilizada que mostra benefícios aos pacientes.

Palavras chave: Anatomia da Coluna Lombar; Lombalgia; Manipulação vertebral. 
The chronical low back pain is continuous pain in the low back region that does not show improvement after three months or more. It has a high prevalence in the population and is one of the main occupational health problems. Manipulation is often used as a treatment, which consists in the application of techniques with the hands, on the patient's body, with the purpose of promoting return to normal function, emphasizing reestablishing joint mobility, promoting analgesia and altering the muscular activity. This article is a review in the scientific literature regarding the technique of manipulation in chronic low back pain. The main databases were searched and considered articles of 2004 so far, as well as books of the area. The studies are controversial, some demonstrate the efficacy in pain relief soon after the application, and some studies have not been satisfactory. Therapy with more sessions seemed to bring benefits. There is the lack of studies with more patients and more professionals involved, however the manipulation is a technique widely used that shows benefits to the patients.

Key words: Anatomy of the Lumbar Spine; Low back pain; Vertebral manipulation.

\section{Introdução}

A lombalgia crônica se refere à dor contínua na região lombo-sacra. Aparece como um tipo frequente de dor crônica, (BARBOSA et al., 2018), onde a coluna vertebral não recuperou seu primitivo equilíbrio biodinâmico, após uma lesão, persistindo a dor além do tempo aceitável para a recuperação, ou ainda pode estar relacionada a processos patológicos crônicos, que provocam dor contínua ou recorrente, por um período de três meses ou mais (MARILISE PIRAN, 2012).

Os distúrbios osteomusculares são um dos principais problemas de saúde ocupacional, sendo a lombalgia um dos principais causadores de morbidade e incapacidade sendo ultrapassada somente pela cefaléia. Sua alta prevalência na população resulta num expressivo impacto socioeconômico negativo por acometer 60 a $80 \%$ da população economicamente ativa, pois aumenta o absenteísmo e diminui a produtividade (JORGE; VIEIRA; SANDOVAL, 2012). Além disso, a dor é uma experiência subjetiva e pessoal, e sua presença na forma crônica traz não apenas mudanças biológicas, mas também mudanças emocionais, cognitivas e comportamentais (BARBOSA et al., 2018). 
Os fatores mecânicos, físicos ou degenerativos são a grande maioria das lombalgias, causadas por processo músculo-esquelético decorrente de esforços, traumas ou microtraumas que causam desarranjos e compressão, distensões músculoligamentares de caráter agudo ou crônico (MARILISE PIRAN, 2012).

A manipulação é umas das técnicas de terapia manual que tem como objetivo a diminuição da dor, correção da postura e das disfunções da coluna vertebral. É um tratamento conservador e não invasivo cujo procedimento é realizado em alta velocidade, pequena amplitude e normalmente no final do movimento.

Recentemente estudos demonstraram que a manipulação promoveu efeitos diretos nos mecanismos neurofisiológicos da dor, assim como na funcionalidade da coluna lombar. (JORGE, 2011).

Tratando-se da lombalgia crônica, uma manipulação poderá garantir o alívio ou eliminação do fator limitante e causador da dor, visto isso, o presente artigo tem como objetivo de revisar os estudos referentes à manipulação na dor lombar crônica.

Esse estudo é de natureza qualitativa e de nível explicativo, na qual foi realizado como pesquisa literária, com sua fundamentação teórica delineada em uma ampla revisão científica.

Foram utilizados livros teóricos da área estudada, juntamente com bases de dados virtuais como Scielo, Pubmed, PEDro, Bireme, LILACS (Literatura LatinoAmericana e do Caribe em Ciências da Saúde), MEDLINE (Literatura Internacional em Ciências da Saúde), BVS (Biblioteca Virtual em Saúde) e DECS (Descritores em Ciências da Saúde).

As buscas nas bases de estudos tiveram como descritores: Anatomia da Coluna Lombar, Lombalgia, Manipulação vertebral. Além disso, foram usados como critérios de inclusão: disponibilidade dos artigos em linguagem portuguesa e inglesa, ter sido publicado a partir do ano de 2004 até a presente data, exceto publicações clássicas do tema.

\section{Anatomia da Coluna Vertebral}


A coluna vertebral é um complexo estrutural cuja principal função é proteger a medula espinhal e transferir cargas entre cabeça e membros (WEIDLE, 2004). É formada por trinta e três vértebras divididas em cinco regiões: cervical, torácica, lombar, sacral e cóccix. Numa vista posterior, a coluna é normalmente vertical. Na vista lateral, a coluna tem quatro curvaturas, duas lordoses e duas cifoses, que vão garantir a maior estabilidade e resistência da coluna vertebral (MENEZES, 2007).

As vértebras lombares têm um corpo vertebral bem maior que as vértebras torácicas. Os pedículos são fortes e dirigidos posteriormente, além das lâminas serem espessas e os foramens vertebrais triangulares. Os processos espinhosos são planos e dirigidos para trás (BEGONHA, 2009). São cinco as vértebras lombares e abaixo delas estão localizados o sacro e o cóccix. Cada uma possui uma porção anterior (corpo vertebral), responsável pela sustentação, suporte de peso e amortecimento de choques e uma porção posterior (orifício), que serve de guia direcional. O alinhamento desses orifícios forma o canal vertebral que serve de passagem para o feixe de nervos da medula espinhal (WATKINS, 2014).

As vértebras são separadas pelos discos intervertebrais, que funcionam como absorvedores de choques. As funções da coluna lombar são: conexão flexível entre as metades superior e inferior do corpo, proteção da medula espinhal, sustentação de peso, flexibilidade e absorção de choques. As estruturas que podem provocar a lombalgia incluem: os ligamentos da coluna, as raízes nervosas e suas meninges, as facetas articulares, o anel fibroso, as articulações facetarias e os músculos profundos. Podem estar presentes alterações estruturais como: vértebra de transição, espinha bífida, escoliose, hiperlordose lombar, retificação da curvatura lombar, espondilolise, espondilolistese, gravidez, postura inadequada, hérnia discal, entre outras (VASCELAI, 2009).

\section{Lombalgia crônica}


Um desequilíbrio mecânico das estruturas da coluna vertebral atua como fator nocivo sobre elas mesmas. Todas as estruturas que compõem a unidade anátomofuncional do segmento lombar apresentam inervação nociceptiva, com exceção do núcleo pulposo e de algumas fibras do anel fibroso (TOSCANO; EGYPTO, 2006). A lombalgia crônica (LC) caracteriza-se por uma dor que perdura após o $3^{\circ}$ mês, a contar do primeiro episódio de dor aguda e, pela instalação da incapacidade, frequentemente tem inicio impreciso e fatores de melhora e piora (MARILISE PIRAN, 2012).

As dificuldades da abordagem e do estudo da dor lombar decorrem da inexistência de uma fidedigna correlação entre os achados clínicos e os de imagem, como também do fato do segmento lombar ser inervado por uma difusa e entrelaçada rede de nervos, tornando difícil determinar com precisão o local e origem da dor, exceto nos acometimentos radículo-medulares (JORGE; VIEIRA; SANDOVAL, 2012).

A lombalgia mecânica comum é a forma mais prevalente e na maioria dos casos se limita à região lombar e nádegas. Raramente se irradia para as coxas. Pode aparecer subitamente pela manhã e apresentar-se acompanhada de escoliose antálgica. O episódio doloroso tem duração média de três a quatro dias. Após esse tempo, o paciente volta à completa normalidade, com ou sem tratamento (BRAZIL et al., 2004).

\section{Efeitos da manipulação na dor lombar crônica}

Segundo RAUSCHKOLB et al, 2016, a manipulação espinhal é um procedimento terapêutico utilizado frequentemente por fisioterapeutas especializados em osteopatia, que utiliza técnicas manuais de mobilização e manipulação articular, massagem do tecido conectivo, fricção transversa, mobilização neural, entre outras, para avaliação e tratamento das dores de origem neuro-músculo-esquelética e de restrição de amplitude de movimentos articulares fisiológicos e acessórios. 
BORTOLAZZO et al. 2015, completa que a manipulação, portanto consiste na aplicação de técnicas com as mãos, sobre o corpo do paciente, com o objetivo de promover o retorno à função normal, destacando-se por reestabelecer a mobilidade articular, promover analgesia e alterar a atividade muscular.

COUTO et al. 2007, diz ainda que a manipulação é um procedimento de terapia manual realizada em alta velocidade, pequena amplitude e normalmente no final de movimento.

Continuando com o autor supracitado, a manipulação parece promover efeitos diretos nos mecanismos neurofisiológicos da dor, assim como, na funcionalidade. Após a aplicação da técnica de manipulação, num período que pode ir de segundos a minutos, é possível observar algum grau de hipoalgesia, pois o sistema descendente noradrenérgico age na medula espinhal e inibe a libertação da substância $\mathrm{P}$, estimulando assim a libertação de opióides endógenos na medula.

Para MOREIRA, 2011, a forma mais comum da manipulação espinhal é um impulso (thrust) de alavanca curta, alta velocidade e de baixa amplitude. A manipulação espinhal por sua própria natureza é uma entrada mecânica aos tecidos da coluna vertebral.

GÓIS, et al, 2006 acrescenta que além de tratamento, também é possível fazer o diagnóstico palpatório de bloqueios tissulares, em geral, e articulares, em particular, igualmente chamados de lesões ou disfunções, utilizando a manipulação

Segundo relatos de MULLER, 2005, no tratamento da dor crônica da coluna essa técnica foi bem mais eficiente, tanto a curto quanto a longo prazo quando comparada com acupuntura e tratamento medicamentoso.

Entrando em conformidade com BICALHO, 2010, onde um estudo com 40 pacientes, após a manipulação houve redução imediata e significativa da dor lombar crônica e melhora na distância dedos-solo.

Em um trabalho feito por FERNANDES et al., 2016, na avaliação dos efeitos de uma intervenção manipulativa sobre a atividade eletromiográfica dos músculos paravertebrais e a intensidade da dor na coluna lombar imediatamente e 30 minutos após sua realização em indivíduos com dor lombar crônica mecânica, quando 
comparado com o grupo controle, que permaneceu em decúbito lateral por dez segundos sobre cada lado do corpo. Foi observada redução significativa na intensidade da dor no grupo que recebeu a manipulação imediatamente e após 30 minutos, ao contrário do grupo controle.

Anteriormente em outro estudo semelhante, YU et al., 2012, também demonstrou que imediatamente após a manipulação, houve a diminuição da atividade elétromiográfica e dor a pressão significativamente em relação ao grupo placebo.

De acordo com COUTO et al. 2007, já o efeito agudo da manipulação articular torácica e manipulação placebo sobre a dor subjetiva e a amplitude de movimento em indivíduos com dor lombar crônica não-específica, onde 16 indivíduos participaram de um estudo clínico duplo-cego, experimental e controlado, a manipulação torácica em pacientes com dor lombar crônica não produziu melhora da dor subjetiva e aumento na amplitude de movimento significativa quando comparado com o tratamento placebo.

Porém, outro estudo clínico realizado por BOND et al., 2019, randomizado e cego, com terapia manipulativa espinhal por três semanas em pacientes com dor lombar crônica não específica, onde foram recrutados 29 participantes e distribuídos aleatoriamente em um grupo que recebeu a terapia de fato $(n=14)$ e outro que recebeu falsamente a terapia $(n=15)$. Foi quantificado o desfecho clínico pré e pós-intervenção, limiar da dor à pressão local, na extremidade inferior e superior, houve a melhora da dor em todos os locais e também diminuição da incapacidade, entretanto esse resultado foi observado nos dois grupos.

De forma similar, CAVALCANTE, 2009 observou que indivíduos que se encontravam em tratamento fisioterapêutico de dores lombares, após avaliação da intensidade da dor, escolheu-se a submissão ao tratamento de terapia manual, através de mobilização articular, durante cinco sessões semanais de dez minutos cada, com posterior avaliação da intensidade álgica. Nos resultados apresentados, houve uma redução significativa ao final de todas as sessões, com o registro de redução quase total dos sintomas de lombalgia crônica ao final das cinco sessões, parecendo que o aumento do número de sessões pode levar a um benefício. 
Na comparação da eficácia das técnicas Maitland, Mulligan e Estabilização Segmentar, cujo parte do tratamento é a manipulação, em relação ao ganho de amplitude de movimento de flexão/extensão de tronco, e diminuição da dor lombar, no tratamento de 21 pacientes com lombalgia crônica, MARILISE PIRAN, 2012, conclui que todos os pacientes dos três grupos obtiveram resultados positivos, apresentaram alívio da dor e melhores resultados ao final do tratamento.

\section{Considerações finais}

Atualmente, o elevado número de pacientes que procuram ambulatórios de fisioterapia traumato-ortopédica com queixas de dor lombar crônica, indica a grande preocupação que se deve ter junto aos mesmos. Para que se tenha um bom diagnóstico cinético-funcional da patologia, o fisioterapeuta deve possuir conhecimentos anatômicos e biomecânicos do local acometido.

Embora ainda existam outras formas de abordagem fisioterapêutica de pacientes com quadro de dor lombar crônica, a técnica de manipulação, têm se mostrado de grande importância e com efeitos positivos na abordagem dos mesmos, restaurando a função articular normal e promovendo um equilíbrio da função muscular. É necessário, entretanto, como em qualquer outro tipo de patologia, que haja sempre um diagnóstico detalhado, para que a intervenção terapêutica através desta técnica seja precisa e eficaz.

Tendo em vista os aspectos observados, mais estudos precisam ser realizados com o intuito de verificar os efeitos mecânicos e neurofisiológicos da manipulação, porém até o momento os pesquisadores, ainda têm dificuldades de quantificar seus achados e principalmente validar os meios diagnósticos que são usados pelos fisioterapeutas, já que o uso da manipulação é extremamente subjetiva.

\section{Referências}


BARBOSA, Fernanda Martins et al. Beliefs and attitudes in patients with chronic low back pain. Brazilian Journal Of Pain, [s. 1.], v. 1, n. 2, p. 116-121, 2018. Disponível em: <http://www.gnresearch.org/doi/10.5935/2595-0118.20180023>. Acesso em: 22 maio. 2019.

BEGONHA, João Botelho Gomes Pereira. Tratamento da dor lombar atrvés do programa de reabilitação $\mathrm{MedX}{ }^{\circledR}$. estágio realizadona clínica Praxis-Centro de Cirurgia Discal Doutor Miguel Enes, [s. 1.], 2009.

BICALHO E, Setti JAP, Macagnan J, Cano JLR, Manffra EF. Immediate effects of a high-velocity spine manipulation in paraspinal muscles activity of nonspecific chronic low-back pain subjects. Man Ther. v. 15 n.5. p.469-75, 2010.

BORTOLAZZO, Gustavo Luiz. Efeitos da manipulação cervical alta sobre a atividadeeletromiográfica dos músculos mastigatórios e amplitude de movimento de abertura da boca em mulheres com disfunção temporomandibular: ensaio clínico randomizado e cego. Fisioter Pesq. v. 22 n.4 p.426-34, 2015.

BOND BM. et al. Effect of spinal manipulative therapy on mechanical pain sensitivity in patients with chronic nonspecific low back pain: a pilot randomized, controlled trial. J Man Manip Ther. v 5 p. 1-13, 2019. Disponível em: https://www.ncbi.nlm.nih.gov/pubmed/30935324. Acesso em 22 maio. 2019

BRAZIL, A. V. et al. Diagnóstico e tratamento das lombalgias e lombociatalgias. Revista Brasileira de Reumatologia, [s. 1.], v. 44, n. 6, p. 419-425, 2004.

CAVALCANTE, E. S. Análise da sintomatologia em indivíduos com lombalgia crônica submetidos à Terapia Manual. Resumo expandido apresentado à Funorte, 2009.

COUTO, I. B. V. L. Efeito agudo da manipulação em pacientes com dor lombar crônica: estudo piloto Fisioterapia em Movimento, v. 20, n. 2, p. 57-62, 2007

FERNANDES, Walkyria Vilas Boas et al. Duração dos efeitos de uma manipulação vertebral sobre a intensidade da dor e atividade eletromiográfica dos paravertebrais de indivíduos com lombalgia crônica mecânica. Fisioterapia e Pesquisa, [s. 1.], v. 23, n. 2, p. 2016. 155-162, Disponível em: <http://www.scielo.br/scielo.php?script=sci_arttext\&pid=S1809-

29502016000200155\&lng=pt\&tlng=pt>. Acesso em: 22 maio. 2019.

GÓIS, R.M. Tratamento da lombalgia crônica através de técnicas alta velocidade baixa amplitude: uma revisão bibliográfica; X Encontro Latino Americano de Iniciação Científica e VI Encontro Latino Americano de Pós-Graduação p.2752-2755; 2006.

JORGE, E. M.; VIEIRA, J. H.; SANDOVAL, R. A. Kinesiology taping nas lombalgias de trabalhadores que atuam na posição sentada. Revista de Transmisión del 
Conocimiento Educativo y de la Salud, [s. 1.], v. 4, n. 3, p. 181-206, 2012.

JORGE, Sílvia Carmen Feliciano. Efeito da manipulação lombar em pacientes com dor lombar aguda. Dissertação Instituto Politécnico do Porto. Escola Superior de Tecnologia da Saúde do Porto, [s. 1.], 2011.

MARILISE PIRAN, Solange Macedo Aily. Análise comparativa do tratamento da dor lombar crônica utilizando-se as técnicas de Maitland, Mulligan e Estabilização Segmentar. EFDeportes.com, Revista Digital. Buenos Aires, [s. 1.], 2012.

MENEZES, Fabio Sprada. Análise cinemática da dinâmica postural do tronco do velejador durante simulação da posição de escora. Dissertação de mestrado, Universidade do Estado de Santa Catarina, [s. 1.], 2007.

MOREIRA, Renata Pessoa. Manipulação osteopática na lombalgia crônica: uma revisão bibliográfica. Disponível em: http://portalbiocursos.com.br/ohs/data/docs/32/131__ManipulaYYo_osteopYtica_na_lombalgia_crYnica_uma_revisYo_bibliogrYfica.pdf.

Acesso em 30 maio. 2019

MULLER, R.; GILES, L.G.F. Long-term followup of a randomized clinical trial assessing the efficacy of medication, acupunture, and spinal manipulation for chronic mechanical spinal pain. Journal of Manipulative and Physiological Therapeutics, $v$. 28, n. 1, pp. 3-11, 2005.

RAUSCHKOLB, P et al. Efeitos das técnicas manuais de mobilização e manipulação articulares da coluna vertebral, revista saúde integrada, v. 9, n. 17, 2016.

TOSCANO, José Jean de Oliveira; EGYPTO, Evandro Pinheiro Do. A influência do sedentarismo na prevalência de lombalgia. Revista Brasileira de Medicina do Esporte, [s. 1.], v. 7, n. 4, p. 132-137, 2006.

VASCELAI, Alessandra. Lombalgias: Mecanismo anátomo-funcional e tratamento. Congresso Sulbrasileiro de DOR, [s. 1.], p. 1-7, 2009.

WATKINS, James. Fundamental Biomechanics of Sport and Exercise. [s.l.] : Routledge, 2014.

WEIDLE, CRISTINE MARIA. O COMPORTAMENTO DA COLUNA VERTEBRAL SOB TRAÇÃO MECÂNICA. 2004. [s. 1.], 2004. Disponível em: <http://www.pilateszone.com.br/wp-content/uploads/2013/12/o-comportamento-dacoluna-vertebral-sob-tracao-mecanica.pdf>

YU, X. et al. Changes in pressure pain thresholds and Basal electromyographic activity after instrumentassisted spinal manipulative therapy in asymptomatic participants: a randomized, controlled trial. Journal of manipulative and physiological therapeutics. v. 35, n. 6. Pág $437-435$. 
SAÚdista
SAL VALES 